\title{
ACTIVE AND POTENTIALLY ACTIVE FORMS OF FIBRIN STABILIZING FACTOR (FACTOR XIII) IN VARIOUS DISORDERS AND IN NEONATAL INFANTS
}

\author{
KANEO YAMADA and ZENZABURO YAMADA \\ Department of Pediatrics, School of Medicine, Keio University, Tokyo Japan
}

(Received for publication April 9, 1968)

Fibrin which is produced from extremely purified fibrinogen with thrombin and calcium ion is soluble in 5M urea or in $1 \%$ monochloroacetic acid (MCA) solution. On the other hand, the one which is produced from fibrinogen or plasma containing FSF (Fibrin Stabilizing Factor) is not soluble in those agents. ${ }^{1-3}$ This fact may be explained as follows: while fibrinogen is polymerized with the weak secondary bond when FSF is excluded, it is polymerized with much stronger covalent bond under the existence of FSF. In this sense, FSF works as cement to tie up fibrin polymers firmly, and then makes fibrin clot stronger. ${ }^{3}$ When this factor is lacking, hemostatic function is depressed and hemorrhagic tendency appears. ${ }^{3}$ Cases in which this factor is congenitally lacking have been reported, and this factor is currently designated as XIIIth factor in blood coagulation

Although FSF is activated by the action of thrombin and calcium ion, a part of this factor seems to remain as a potentially active form in plasma even with sufficient thrombin and calcium ion. This potentially active form is activated in vitro by the addition of agents containing sulfhydryl moiety such as cysteine or glutathione. Nussbaum and Morse ${ }^{ \pm}$determined FSF values in various adult cases, and found that low values of FSF were mostly due to the fact that a larger part of this factor remained as a potentially active form and it was restored to normal level in many cases by the addition of cysteine.

We have already reported on FSF values especially in children under various developmental stages and with pathological conditions. ${ }^{5}$ Our study revealed that FSF value stayed low through the first week after birth. And in pathological group, low FSF value was found in the cases of acquired hypofibrinogenemia, liver disease, leukemia, idiopathic thrombocytopenic purpura etc. 
In this study we have examined FSF values of plasma in vitro with and without cysteine, on cases which were characterized by low value of FSF.

\section{CASES}

Authors classified materials into five groups. The first group consists of healthy adults, preschool and school children; the second one of normal fullterm infants within 7 days after birth; the third one of liver diseases such as congenital atresia of the bile ducts, cystic dilatation of the common bile ducts, hepatitis etc.; the forth one of Kasabach-Merritt syndrome, promyelocytic leukemia and other defibrination syndrome occured in the course of viral infection. Third group was further divided into two groups, i.e. the one indicating extremely low value and the other staying within normal range. Their platelet count were within normal limit in both group. In the fourth group,hypofibrinogenemia was associated with the decrease in platelet count which went down below 20,000. This group may be placed under the category of consumption coagulopathy.

The fifth group consists of various other diseases in childhood other than the third and forth groups.

\section{METHOD}

Sample: One part of $3.8 \%$ sodium citrate solution was added to nine parts of blood as soon as it was withdrawn, and centrifuged at $3000 \mathrm{rpm}$ for 15 minutes at $0^{\circ} \mathrm{C}$. Plasma was stored frozen and used within one week.

Tris buffer solution: Tris $0.05 \mathrm{M}$, sodium chloride $0.1 \mathrm{M}, \mathrm{pH} \mathbf{7 . 5 5} \pm \mathbf{0 . 1}$.

FSF free fibrinogen ( $1 \%$ solution in tris buffer): $: 6,7$ One percent saline solution of fibrinogen (Armour fraction \#I) was salted out twice with $25 \%$ saturated ammonium sulfate solution, and this precipitate was prepared to $2 \%$ solution with tris buffer. This solution was dialysed against tris buffer and finally adjusted to $1 \%$ solution.

In order to destroy FSF, freezing and thawing were repeated three times at $-20^{\circ} \mathrm{C}$ and $+38^{\circ} \mathrm{C}$. This fibrinogen solution was tested for the existence of FSF by adding tris buffer, thrombin and calcium ion. The clot thus formed was dissolved immediately in $2 \%$ MCA. When a clot was formed under the existence of cysteine, it was also dissolved within about ten minutes. From these observations, our fibrinogen was regarded as almost FSF free. In order to determine FSF value, plasma was treated by the procedure as described in table 1 , and the amount of potential active FSF was roughly estimated by substract- 
ing the value obtained by this procedure from the value obtained by the procedure as described in table 2 which transforms the potential active form to the active form by adding cysteine. The dissolution of fibrin clot was checked after 18 hours and the degree of dissolution was classified in the descending order as $-,+, H, H, \ldots+$ depending on the amount of undissolved fibrin clot.

Table 1

\begin{tabular}{|c|c|c|c|c|}
\hline plasma & 0.1 & 0.2 & 0.4 & $0.8 \mathrm{ml}$ \\
\hline tris buffer (pH 7.55) & 0.8 & 0.7 & 0.5 & 0.1 \\
\hline $1 \%$ fibrinogen FSF $(-)$ & 0.5 & $" \prime$ & $n$ & " \\
\hline tris buffer (pH 7.55) & 0.5 & $\prime \prime$ & $" \prime$ & $\prime \prime$ \\
\hline $0.01 \mathrm{M} \mathrm{CaCl}_{2}$ & 0.5 & $" \prime$ & " & $" \prime$ \\
\hline \multirow[t]{5}{*}{ 20u. thrombin } & 0.1 & $" \prime$ & " & $"$ \\
\hline & & & \multicolumn{2}{|c|}{$37^{\circ}, 30 \mathrm{~min}}$. \\
\hline & \multicolumn{4}{|c|}{ fibrin clot } \\
\hline & & & \multicolumn{2}{|c|}{$\begin{array}{l}2.5 \mathrm{ml} \text { monochloro- } \\
\text { acetic acid, } 2 \%\end{array}$} \\
\hline & \multicolumn{4}{|c|}{ check the solubility of fibrin after 18 hours } \\
\hline
\end{tabular}

Table 2

\begin{tabular}{|c|c|c|c|c|}
\hline plasma & 0.1 & 0.2 & 0.4 & $0.8 \mathrm{ml}$ \\
\hline tris buffer (pH 7.55) & 0.8 & 0.7 & 0.5 & 0.1 \\
\hline $1 \%$ fibrinogen FSF $(-)$ & 0.5 & $" \prime$ & $" \prime$ & $\prime \prime$ \\
\hline $0.1 \mathrm{M}$ cysteine ( $\mathrm{pH} 7.55)$ & 0.5 & $\prime \prime$ & $" \prime$ & $\prime \prime$ \\
\hline $0.01 \mathrm{M} \mathrm{CaCl}_{2}$ & 0.5 & $\prime \prime$ & $\prime \prime$ & $" \prime$ \\
\hline \multirow[t]{5}{*}{ 20u. thrombin } & 0.1 & $" \prime$ & " & $"$ \\
\hline & & & \multicolumn{2}{|c|}{$37^{\circ}, 30 \mathrm{~min}}$. \\
\hline & & \multicolumn{3}{|c|}{ fibrin clot } \\
\hline & & & \multicolumn{2}{|c|}{$\begin{array}{l}2.5 \mathrm{ml} \text { monochloro- } \\
\text { acetic acid, } 2 \%\end{array}$} \\
\hline & leck th & olubil & of $\mathrm{fit}$ & after 18 hours \\
\hline
\end{tabular}

As we reported previously, the fibrin clot of healthy adults and children (the first group) remained undissolved when $0.1 \mathrm{ml}$ of plasma was used, however it was completely dissolved when the amount of plasma was decreased down to 
$0.075 \mathrm{ml}$. In this paper, the plasma corresponding to this activity was assumed to contain $100 \%$ of FSF. When the clot was dissolved with plasma of down to $a$ $\mathrm{ml}$, the FSF value of the sample would be expressed as $0.10 / \mathrm{a} \times 100 \%$.

The amount of fibrinogen was determined by tyrosine method or weighing method.

\section{RESULTS}

1. An attempt to examine the correlation between FSF value and the amount of fibrinogen was made prior to the experiment on the effect of cysteine on FSF value.

a) The results on normal adults and children, and full term infants, as classified the first and the second groups respectively, are shown in Fig. 1. As reported previously, FSF value in newborn period is generally less than $50 \%$ of that of the normal adults and children in more than a half of the cases. However, the amount of fibrinogen remained normal even in those cases with physiological low value of FSF.

b) In 5 cases of liver disease, the level of fibrinogen was generally low, and 3 cases out of five showed low FSF value of less than 50\% (Fig. 2).

All cases of consumption coagulopathy had the level of fibrinogen less than $125 \mathrm{mg} / \mathrm{dl}$, and also had very low FSF value of less than $25 \%$ (Fig. 3 ).

c) In various diseases other than the above, slight but not marked correlation was observed between the level of fibrinogen and FSF value (Fig. 4).

These results seem to indicate that very low level of fibrinogen in cases such as consumption coagulopathy and liver diseases is usually attended by low level of FSF. In order to analyze the cause of low level of FSF in these diseases, effect of addition of cysteine to plasma from these cases was explored.

2. Alteration in FSF value by the addition of cysteine.

a) In the first group consisting of healthy adults and infants, the level of FSF of $100 \%$ was elevated up to $150 \%$ by the addition of cysteine, although some still remained at $100 \%$. Only one case in this group showing $50 \%$, was elevated up to $100 \%$ (Fig. 5).

b) In cases of full term newborns, two cases showing $33 \%$ were raised up to $50 \%$ and one case showing $50 \%$ raised up to $100 \%$ by the addition of eysteine, and thus the elevation by this procedure was only partial (Fig. 6).

c) In the third group of liver diseases, two cases of $25 \%$ were improved up to $100 \%$, and one case of $33 \%$ was raised up to $150 \%$ both by the addition of cysteine. This group showed the most remarkable elevation among others by 


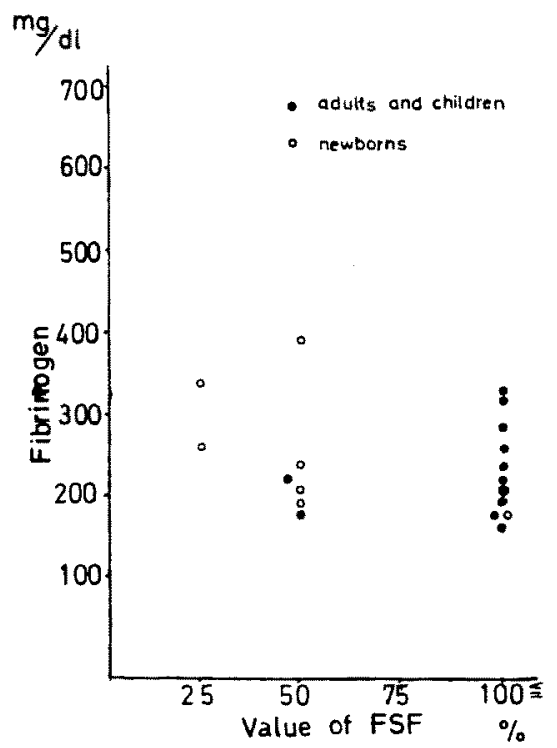

Fig. 1

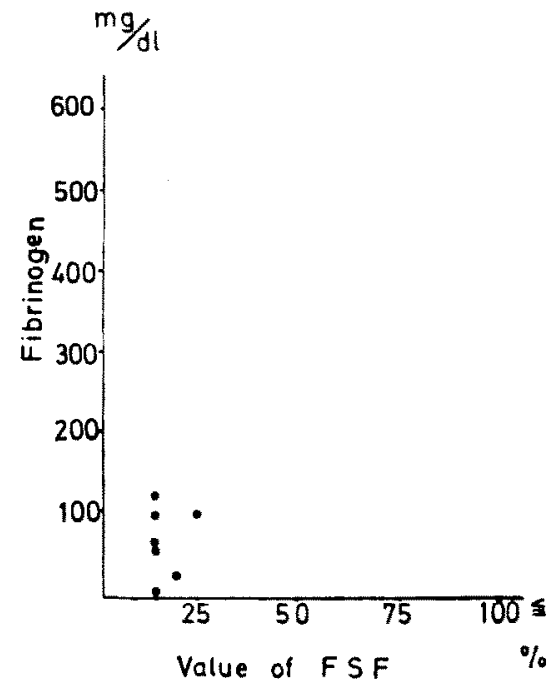

Fig. 3

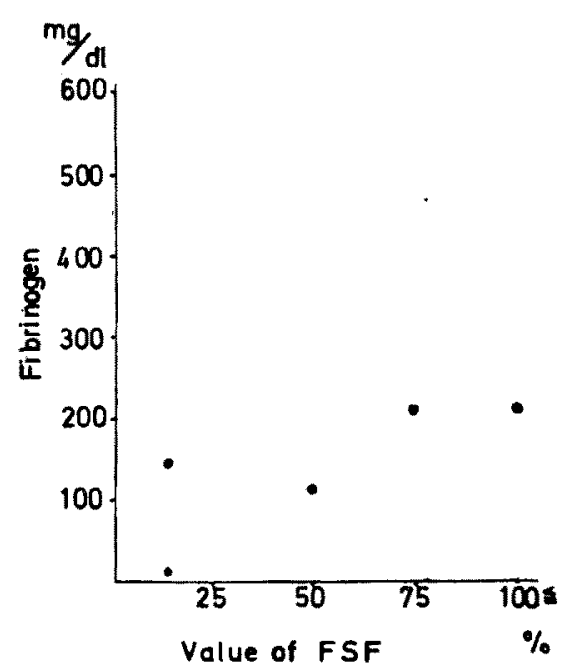

Fig. 2

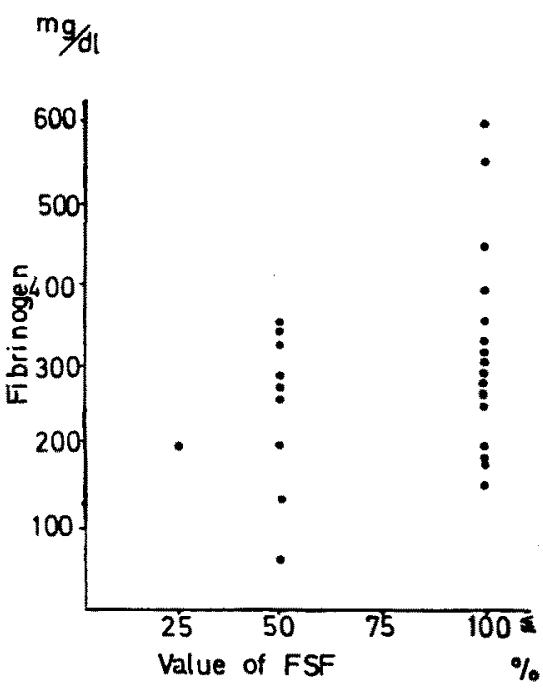

Fig. 4

Fig. 1 Relationship between the amount of fibrinogen and the value of FSF in normal adults and children, and newborn infants.

Fig. 2 Relationship between the amount of fibrinogen and the value of FSF in liver diseases.

Fig. 3 Relationship between the amount of fibrinogen and the value of FSF in consumption coagulopathies.

Fig. 4 Relationship between the amount of fibrinogen and the value of FSF in various diseases. 


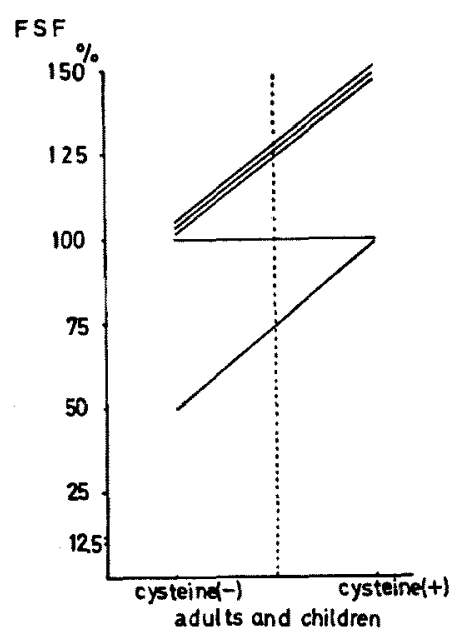

Fig. 5

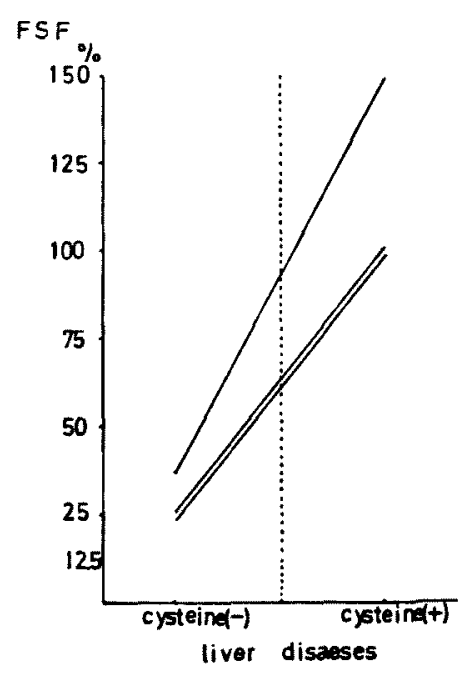

Fig. 7

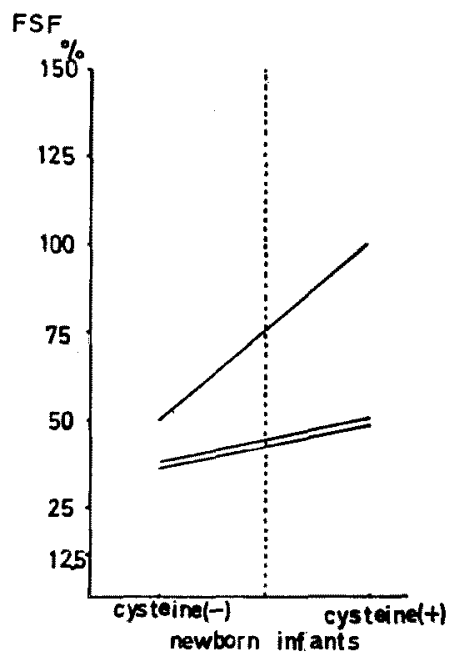

Fig. 6

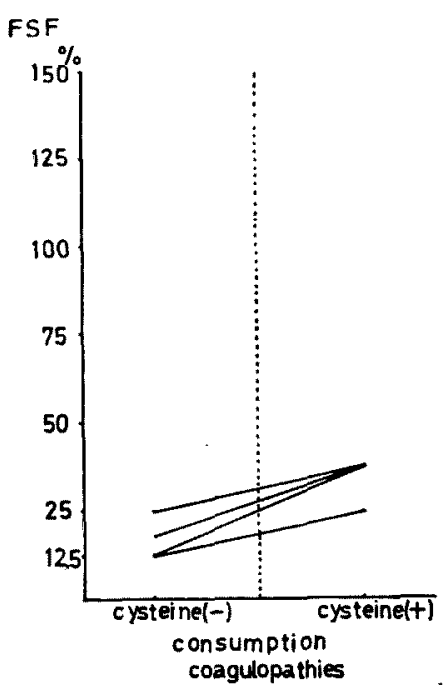

Fig. 8

Fig. 5 Alteration of the value of FSF in adults and children by the addition of cysteine.

Fig. 6 Alteration of the value of FSF in newborn infants by the addition of cysteine.

Fig. 7 Alteration of the value of FSF in liver diseases by the addition of cysteine.

Fig. 8 Alteration of the value of FSF in consumption coagulopathies by the addition of cysteine. 
the addition of cysteine (Fig. 7).

d) In the fourth group consisting of consumption coagulopathy, three cases showing 12.5-25\% were elevated to $33.3 \%$ and one case of $12.5 \%$ to $25 \%$. In this group, the effect of cysteine to raise FSF value was the smallest (Fig. 8).

\section{COMMENTS}

Cysteine and glutathione, both having sulfhydryl group, are known to activate FSF in vitro. Lowy and Edsall ${ }^{8}$ have found the fact that the fibrin clot formed with cysteine or with glutathione did not dissolve in 5M urea solution, or in 1\% MCA solution, even when highly purified fibrinogen with least contamination of FSF was used. This fact seems to suggest the possibility that cysteine or glutathione is compensating the action of FSF.

However, Lorand and Jacobson" were not agreeable with this hypothesis, and they further carried out the experiment and found fibrin clot with cysteine is no more insoluble, when the highly purified fibrinogen, incubated at $40^{\circ} \mathrm{C}$ for 3 hours prior to the fibrin formation, was used.

They have concluded from these results that cysteine or glutathione can not substitute FSF action but merely activate its potentially active form to an active one.

Based on this theory, we now are able to measure the amount of the active form and the potentially active form of FSF separately, by examining solubility of fibrin clot in MCA without or with cysteine.

Nussbaum and Morse ${ }^{4}$ determined FSF value in adults with various diseases. They reported that 85 out of 95 cases with low FSF value restored its value to normal by the addition of eysteine or other sulfhydryl compounds in vitro.

However, 6 cases of leukemia, 3 cases of severe hypofibrinogenemia and one case of ITP did not restore its activity even by the addition of cysteine.

We further measured these two forms of FSF on consumption coagulopathy and liver diseases. In our study, low level of FSF was not restored to normal by the addition of cysteine in the case of consumption coagulopathy such as Kasabach-Merritt syndrome, promyelocytic leukemia, and other defibrination syndrome. On the contrary, it was restored to normal in most cases of liver diseases.

This low values of FSF and the restoration of FSF activity by the addition of cysteine in liver disease can be explained by assuming that, FSF is existing in the plasma of patients with liver diseases mostly as potentially active form. 
This may suggest the deficiency of substance which converts potentially active form of FSF to the active form in liver diseases. It also suggests that the production of potentially active form is not necessarilly inhibited even in cases where the production of fibrinogen is deficient in liver diseases.

On the other hand, FSF seems to be consumed together with platelets, fibrinogen, the Vth and VIIIth factors, and prothrombin in case of consumption coagulopathy, and thus potentially active form is also decreased beside the deficiency of active form.

In cases of newborn infants, the restoration of low value of FSF by the addition of cysteine is only partial. Levels of both forms of FSF in newborn cases are rather low. This fact may indicate the immaturity of the newborn infants in this respect.

\section{SUMMARY}

1) Correlation between the amount of fibrinogen and value of FSF in plasma obtained from normal adults and children, newborn infants, and also in various disorders was examined.

In the cases of hypofibrinogenemia showing less than $100 \mathrm{mg} / \mathrm{dl}$, the value of FSF decreased to the level less than $25 \%$. These remarkable cases consisted of consumption coagulopathy such as Kasabach-Merritt syndrome, promyelocytic leukemia etc. and liver diseases such as congenital atresia of bile ducts, cystic dilatation of common bile ducts, hepatitis etc.

In spite of the strong correlation between these two factors in the extreme cases of pathological group it was also noted that the amount of fibrinogen remained normal in the cases of newborn infants showing physiological low level of FSF.

2) Alteration in FSF value by the addition of cysteine in vitro was observed.

a) In consumption coagulopathy, the value of FSF could not be restored by addition of cysteine.

b) In liver diseases, the low value of FSF restored to the normal by cysteine.

c) In newborn infants, although decreased values of FSF elevated to some extent by the addition of cysteine, their restoration did not reach to the level of healthy adults and children.

Based on our findings, it is likely that, FSF in liver diseases exist mostly as a potentially active form, and in consumption coagulopathy, both active and 
potentially active forms have been consumed. In neonatal period, the production of these two forms are at the immature stage.

\section{REFERENCE}

1. Laki, K. \& Lorand, L.: On the solubility of fibrin clots. Science, 108: 280, 1948.

2. Lorand, L.: Fibrin clot. Nature, 166: 694-695, 1950.

3. Ferry, J. D., Miller, M. \& Shulman, S.: The conversion of fibrinogen to fibrin. VII. Rigidity and stress relaxation of fibrin clots; effect of calcium. Arch. Biochem. \& Biophys., 34: 424-436, 1951.

4. Nussbaum, M \& Morse, R. S.: Plasma fibrin stabilizing factor activity in various disease. Blood, 23: 669-677, 1964.

5. Yamada, K., Yamada, Z., Tsuchiya, Y., and Noguchi, K.: Fibrin stabilizing factor (factor XIII) in children. Jap. J. Clin. Hemat., 9: 22-28, 1968. (in Japanese)

6. Lorand, L.: In Blood coagulation, hemorrhage and thrombosis. Method of study, edited by L. M. Tocantis and L. A. Kazal. New York, Grune \& Stratton, 1964, p. 239 .

7. Lorand, L. and Dickenman, F.: Assay method for the "Fibrin-Stabilizing Factor." (21711). Proc. Soc. Exp. Biol. Med., 89: 45-48, 1955.

8. Lowy, A. G. \& Edsall, J. T.: Studies on the formation of urea-insoluble fibrin. J. Biol. Chem., 211: 829-839, 1954.

9. Lorand, L. \& Jacobsen, A.: Studies on polymerization of fibrin: Role of globulin: fibrin-stabilizing factor. J. Biol. Chem., 230: 421-434, 1958. 